\begin{tabular}{|c|c|c|c|c|}
\hline Revista Praxis & ISSN: $1657-4915$ & Vol. 12 & $78-89$ & Enero - Diciembre de 2016 \\
\hline \multicolumn{3}{|c|}{ DOI: http://dx.doi.org/10.21676/23897856.1849 }
\end{tabular}

\title{
COMPETENCIAS DOCENTES: DESDE UNA PERSPECTIVA ETNOEDUCATIVA Y TECNOLÓGICA
}

\section{TEACHER'S COMPETENCIES: FROM A TECHNOLOGICAL AND ETHNO-EDUCATIVE PERSPECTIVE}

\author{
Nancy Rosa Roys-Romero ${ }^{1}$
}

\begin{abstract}
RESUMEN
El desarrollo y fortalecimiento de competencias docentes en los estudiantes en formación del Programa de Licenciatura en Etnoeducación de la Universidad de la Guajira requiere de un proceso innovador que permita identificar las competencias que más se adapten al entorno y al contexto, para después con ayuda de la tecnología diseñar e implementar un AVA para el fortalecimiento de dichas competencias. La metodología de investigación se realizó bajo un paradigma cualitativo y un diseño de Investigación Acción Educativa (IAE), a una muestra de 78 estudiantes. Los resultados muestran los beneficios de la implementación del AVA para desarrollar competencias docentes que les brinden conocimientos necesarios a los estudiantes en formación para que desde una perspectiva etnoeducativa obtengan una educación de calidad.
\end{abstract}

Palabras clave: Competencias Docentes, Etnoeducación, Diversidad, Tecnología, AVA.

\begin{abstract}
The development and strength of teacher's competencies in training students in the Ethno-education Program from La Guajira University requires innovative process that allows to identify the competencies that best suit the environment and place and then with the use of technology design and use an AVA on developing these skills. The research methodology was conducted under a qualitative paradigm and an Educational Action Research design (IAE), in a test conducted on 78 students. The results show the benefits of the implementation of AVA to develop teaching competencies that provides students the necessary knowledgably skills from an ethnic education perspective for a quality education.
\end{abstract}

Keywords: Teaching competencies, Ethnic Education, Diversity, Technology, AVA.

Fecha de recepción: Julio 28 de 2015 / Fecha de aceptación: Noviembre 13 de 2015

Tipología: Artículo de Investigación Científica y Tecnológica

Para citar este artículo: Roys, R. N. (2016). Competencias docentes: Desde una perspectiva Etnoeducativa y Tecnológica. Praxis. Vol. $12,78-89$

1. Magistra en E-learning. Universidad de La Guajira, Colombia. Email: nrosaroys@uniguajira.edu.co 


\section{INTRODUCCIÓN}

$\mathrm{L}$ as competencias docentes son de suma importancia para la buena ejecución del quehacer pedagógico, por lo que son en sí mismas un desafío y el sentido de la enseñanza en las facultades de educación, de modo que los parámetros para la renovación curricular se erigen en torno a ellas. Renovación que se hace con el fin de que el proceso académico a desarrollar por parte de los estudiantes este acorde con las exigencias sociales y culturales, asunto que en la actualidad está altamente relacionado con la tecnología como complemento en procura del desarrollo de aprendizaje y de la renovación continua de nuevas habilidades y destrezas.

Lo anterior se enfrenta a la realidad que vive el programa de Licenciatura en Etnoeducación de la Universidad de La Guajira, el cual tiene un enfoque basado en la diversidad étnica e intercultural, cuenta con una alta población de estudiantes pertenecientes a la etnia wayuu, quienes laboran como docentes en Centros Etnoeducativos y en aulas -comunidad en el departamento, quienes al enfrentarse a la vinculación de las tecnologías como herramientas para el ejercicio docente tienen un choque en dos sentidos, uno epistemológico y otro social. El primero radica en que las concepciones de enseñanza, de aprendizaje y de sujeto que aprende en su etnia, dista de la instrumentalización implícita en el uso de tecnologías y el segundo, en que el acceso a las tecnologías en las comunidades donde labora es limitado e incluso es ajeno a los modos de enseñar y aprender en ellas.

No obstante, los maestros en formación del programa mencionado no dejan de estar en contacto con las tecnologías y con el desarrollo de competencias desde una visión occidental de la pedagogía, pues en la Licenciatura en Etnoeducación se propicia este contacto en el marco de la orientación profesional, de allí que, en función de mirar cómo están las competencias de ellos, en esta investigación se hace necesario un diagnostico general sobre el manejo de competencias, se continua con la elaboración de un marco teórico sobre las diversas competencias docentes existentes que nos sirven como referencias para realizar un comparativo de cuales de ellas se adaptan al contexto etnoeducativo y proponer las competencias que se requieran para un perfeccionamiento del proceso educativo e integral de los estudiantes. Luego se propone fortalecer las competencias a través de un Ambiente Virtual de Aprendizaje (AVA), teniendo en cuanta las herramientas interactivas ofrecidas por las TIC.

Los docentes hoy en día se ven enfrentados a nuevos contextos educativos que plantean nuevos desafíos y exigencias, tomando en cuenta la siguiente afirmación nos preguntamos lo siguiente: ¿Los estudiantes que se están formando en el programa cuentan con las competencias necesarias que debe tener un etnoeducador para su quehacer pedagógico? Para su análisis, se observó que los estudiantes en formación del programa han presentado bajos puntajes en las pruebas SABER PRO (2012-2013) que miden el grado de desarrollo de las competencias genéricas y específicas de los mismos, este bajo nivel se debe al manejo incorrecto de las competencias desde el currículo y desde el desarrollo de las mismas en el aula de clases, lo que genera una educación de baja calidad que repercute en los estudiantes. tanto en su formación integral, como en sus saberes.

A continuación se muestra el análisis de las pruebas SABER PRO (2012-2013) presentadas por los estudiantes del Programa; en el caso de las competencias genéricas se ha evidenciado un bajo desempeño en los módulos que la componen, sin descuidar que en algunos de los mismos, el comportamiento ha sido regular; esto se sustenta en el hecho de que su comportamiento ha sido inferior o relativamente inferior al Promedio Nacional, es decir, si éste es de 10,1 que de por cierto es regular, el hecho de que los estudiantes del programa estén por debajo del mismo, permite afirmar que hay un bajo rendimiento en las cohortes señaladas. 
Figura.1.

Promedio por periodo de las Competencias Genéricas

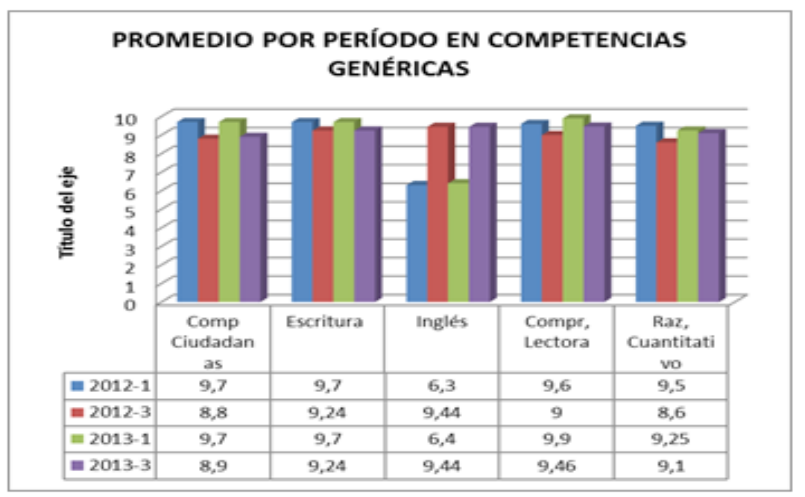

Fuente: Elaboración Propia

En cuanto a las competencias específicas, los estudiantes se ubicaron por debajo de la media nacional que fue de 10.1; con un rendimiento regular en los módulos formar, evaluar, enseñar y la combinación enseñar, formar y evaluar.

\section{Figura.2.}

Promedio por periodo en Competencias Específicas

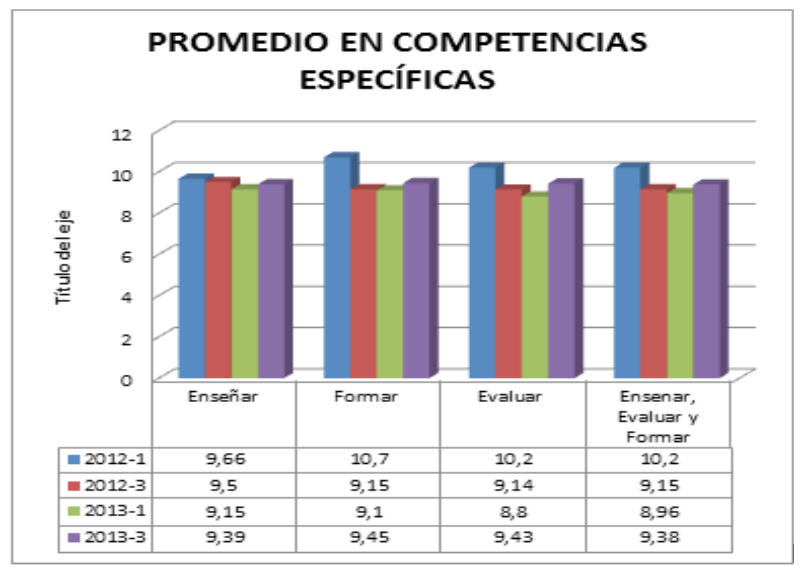

Fuente: Elaboración Propia

De seguir esta situación, los estudiantes en formación continuarán con bajos niveles de aprendizaje y de competencias, por lo tanto, esto se verá reflejado en su proceso formativo y laboral, por ende en la calidad y el desarrollo de nuevas experiencias en la forma de aprender y enseñar de manera significativa e innovadora.

De lo expuesto anteriormente, se desea profundizar sobre las diferentes competencias docentes para poder abordarlas desde diferentes escenarios y así contribuir a la práctica pedagógica; además, se propone transformar las prácticas educativas en relación a la formación de competencias docentes con apoyo de un ambiente virtual de aprendizaje a través del uso de las herramientas web 2.0, donde los maestros en formación puedan mejorar su desarrollo y práctica profesional de una manera innovadora y participativa. Para dar soporte a esta búsqueda se tiene en cuenta la opinión de Velásquez \& López (2011) que afirma que el objetivo primordial de la educación científica y tecnológica es formar a los estudiantes en competencias que les permitan moverse en un mundo impregnado por los avances científicos y tecnológicos, de tal manera que contribuya a la apropiación de los conocimientos... (p.43). Es necesario que la educación científica y tecnológica que se imparta sea de calidad en todos los estratos sociales, y que su enseñanza considere no solo conocimientos y habilidades (p. 44).

En relación con la temática de competencias docentes enfocadas a la labor de un etnoeducador, se estructura el siguiente proyecto desde dos perspectivas, la educativa y la tecnológica, con el objetivo de diseñar un AVA para fortalecer las competencias.

Empecemos por definir el concepto de competencias. Veamos algunos autores y sus definiciones:

Según MEN (2006), "una competencia es entendida como saber hacer en situaciones concretas que requieren la aplicación creativa, flexible y responsable de conocimientos, habilidades y actitudes” (p.12).

Perrenoud (2011), aborda la profesión docente de una manera concreta redefiniendo la profesionalidad del docente; para ello toma como guía el referencial de competencias adoptado en Ginebra en 1996, en donde el concepto de competencia representa la capacidad de movilizar varios recursos cognitivos para hacer frente a un tipo de situaciones; además, se considera como un saber hacer de alto nivel, que permite afrontar una serie de problemas y buscar soluciones adaptadas al contexto.

De Ketele (1996) (citado por Méndez, 2009) las asume como: 
un conjunto ordenado de capacidades que se ejercen sobre los contenidos de aprendizaje y cuya integración permite resolver los problemas que se plantean dentro de una categoría de situaciones y que en relación al concepto de "situación" éste evoca a un conjunto contextualizado de informaciones que un alumno o grupo de alumnos, deberá articular a fin de resolver una tarea determinada. Si dicha situación presenta un obstáculo, cuya solución permitirá nuevos aprendizajes, podemos hablar de "Situación-Problema", están orientadas a integrar los aprendizajes con los saberes. (p. 2)

Según Zabalza \& Arnau (2008), las competencias se definen como la capacidad o habilidad de efectuar tareas o hacer frente a situaciones diversas de forma eficaz en un contexto determinado, y para ello necesario movilizar actitudes, habilidades y conocimientos al mismo tiempo y de forma interrelacionada (p. 43-44).

Al indagar sobre el concepto de competencias docentes desde una perspectiva etnoeducativa se evidencia una nula información al respecto, esa perspectiva no existe, razón por la cual se busca un acercamiento específico, ya que en el Departamento de La Guajira existen Instituciones Etnoeducativas en las que sus docentes, llamados etnoeducadores, son quienes orientan y facilitan el proceso académico de sus estudiantes, proceso fundamentado en la diversidad de los grupos étnicos y de fortalecer la cultura de los contextos internos y externos. Estas competencias están enfocadas a contextos étnicos e inter y multiculturales donde se prioriza las necesidades y expectativas de los mismos hacia una educación de calidad respetando la cosmovisión, costumbres e idioma que prevaleciente en la región y brindando una dimensión para la adquisición de habilidades y experiencias que apoyen al estudiante.

Desde la perspectiva tecnológica, iniciamos con la definición de ambiente virtual de aprendizaje:

Un AVA es un sistema de herramientas de interrelación basado en páginas web e internet, que tienen como finalidad la conformación de comunidades virtuales para apoyar actividades educativas presenciales y como la principal estrategia en la organización e implantación de cursos en línea (López, 2013, p.100).
Según Boneu (2007) los AVA tienen 4 características básicas, estas son:

- Interactividad. Conseguir que la persona que está usando la plataforma tenga conciencia de que es el protagonista de su formación.

- Flexibilidad. Conjunto de funcionalidades que permiten que el sistema de e-learning tenga una adaptación fácil en la organización donde se quiera implementar.

- Escalabilidad. Capacidad de la plataforma de e-learning de funcionar igualmente con un número pequeño o grande de usuarios,

- Estandarización. Capacidad de utilizar cursos realizados por terceros en otros estándares. (p.40)

En relación con las investigaciones que sirven de soporte para el desarrollo de esta investigación, encontramos aportes que se desarrollan con la tecnología y que contribuyen a formar competencias en los docentes. A continuación, se describen los más significativos:

A nivel Nacional se encuentra el proyecto de investigación titulado "Programa de computador para el desarrollo de competencias docentes", el cual es la base de un libro titulado Formación de competencias docentes. Diseñar y aprender con ambientes computacionales y que fue realizado por docentes para estudiantes de licenciaturas en la Universidad Pedagógica Nacional de la ciudad de Bogotá.

Sanabria \&Macías (2006) soportan esta investigación en un análisis cualitativo basado en el desarrollo de competencias cognitivas, metacognitivas y tecnológicas que contribuyen a la formación docente, y a su vez, garantizan un aprendizaje significativo, con la incorporación de las TIC.

Otro proyecto muy significativo en el contexto nacional es el de Ardila (2009) de la Universidad Pedagógica Nacional llamado "Competencias docentes y calidad de la educación superior en ambientes virtuales”. Esta investigación de carácter exploratorio-descriptivo tiene como finalidad identificar, desarrollar y evaluar competencias docentes a través de los ambientes virtuales. El proyecto toma como punto de partida el concepto de competencia de Claude (1996) éste es: "Las competencias hacen referencia a las capacidades para realizar roles y situaciones de trabajo a los 
niveles requeridos en el empleo." El proyecto incluyó una muestra de 786 docentes de 3 universidades colombianas de carácter privado y oficial y dicho estudio posibilitó conocer las competencias docentes más importantes y que permitan realizar procesos de comunicaciones, asesorías, seguimiento y acompañamiento a los estudiantes y establecer relaciones de calidad en la Educación Superior.

El aporte de este proyecto de investigación consiste en el análisis que se hace con las competencias docentes a partir de situaciones de trabajo y aprendizaje con ayuda de la tecnología.

A nivel regional encontramos a Ricardo (2013), con su tesis doctoral titulada "Formación y desarrollo de la competencia intercultural en AVA" cuyo objetivo fue general diseñar, implementar y evaluar una propuesta pedagógica para el desarrollo de la competencia intercultural en AVA. En esta investigación se identifican 3 fases básicas: estudio diagnóstico, diseño e implementación de la propuesta de formación y el análisis y evaluación de la práctica educativa en AVA. El proyecto fue realizado en la Costa Caribe Colombiana donde existe una amplia diversidad cultural, se seleccionaron 10 Instituciones de Educación Superior de la Costa Caribe: CECAR, Universidad de La Guajira, CUC, Universidad Tecnológica de Bolívar, Universidad Autónoma del Caribe, Universidad Popular del Cesar, Universidad del Norte y la Universidad del Magdalena.

Su aporte consistió, en primer lugar, en proponer el uso de los ambientes virtuales de aprendizaje como una manera de facilitar el acceso a materiales de aprendizaje; en segundo lugar, en dar a entender que los espacios de interacción permiten fundamentar relaciones, valores, vivencias que tienen como objetivos el aprendizaje, las actividades, los roles y el uso de la tecnología. Por último, consistió en argumentar que con la educación virtual se recrean situaciones didácticas centradas en el estudiante y que promueven procesos de innovación y el desarrollo de estrategias activas, participativas y constructivistas.

Otro proyecto significativo en el tema es titulado "Una educación para el desarrollo de competencias" fue realizado en la ciudad de Riohacha, La Guajira (Díaz Osorio, Gutiérrez, Merlano y
Díaz Morales, 2010), donde se realizó un análisis del concepto de competencias, además se analizó los criterios, procesos y estrategias que deben seguir las instituciones educativas; por otra parte, se describió una metodología para definir competencias específicas y sus modalidades. Las competencias que tienen en cuenta para el desarrollo del documento investigativo es tomado del Ministerio de Educación Nacional y son las competencias funcionales y comportamentales. Las funcionales corresponden al desempeño de las responsabilidades específicas de los docentes o directivos docentes y las comportamentales se refieren a actitudes, los valores, los intereses y las motivaciones docentes.

Para realizar el trabajo por competencias se utilizan tres metodologías: trabajo por proyectos, resolución de problemas y enseñanza para la comprensión.

Este proyecto concluye que la implantación de la formación por competencia demanda un proceso de transformación radical de un paradigma educativo; además implica el cambio en la manera de hacer docencia. Su aporte muestra que las competencias se aprenden cuando se actúa en cualquier contexto o situación, para comprender el propósito pedagógico y que desarrolle en los docentes, metodologías y estrategias propias de su disciplina adaptadas a una realidad cambiante.

\section{METODOLOGÍA}

La investigación se realizó bajo el paradigma cualitativo, puesto que desde este abordaje se buscó establecer cuáles son las diferentes ópticas y realidades que se han desarrollado frente al tema de competencias docentes y así comprender la información sobre la misma.

En relación con el diseño metodológico se empleó una investigación aplicada, la cual busca comprender e interpretar los conocimientos para mejorar la calidad educativa o descubrir un nuevo conocimiento a través de la población participante donde integra la teoría y la práctica para acercarla a la realidad contextual, donde se investiga desde una nueva óptica las competencias docentes que deben tener los etnoeducadores y fortalecerlas a partir de un AVA con ayuda de herramientas tecnológicas. 
Para el desarrollo de la investigación se tomó como población a los estudiantes del Programa de Licenciatura en Etnoeducación para la básica primaria de la Universidad de La Guajira. En la actualidad el programa cuenta con un total de 259 estudiantes en modalidad presencial y 145 en modalidad de educación a distancia.

La muestra obtenida fue de 78 estudiantes del Programa de Licenciatura en Etnoeducación de ambas modalidades. Se implementó una metodología de investigación acción educativa que busca mejorar la calidad de los procesos educativos y profesionales. Para la recolección de información se aplicó un cuestionario con el objetivo de conocer el nivel de importancia y el nivel de desarrollo que ha alcanzado el estudiante durante sus estudios en el tema de competencias docentes. Al final del curso se aplicó otro cuestionario para recoger las apreciaciones sobre la evaluación general del AVA y las competencias que más fortalecieron los estudiantes.

Las actividades que se realizaron fueron las siguientes:

- Recolección y Análisis de Información sobre competencias docentes.

- Diagnóstico sobre teóricos y proyectos relacionados con las competencias docentes.

- Selección de la metodología de trabajo en línea para diseño del AVA.
- Diseño y aplicación del Instrumento Inicial.

- Tabulación y Análisis de Resultados.

- Diseño del AVA y del Plan docente.

- Diseño y aplicación del Instrumento final sobre la Evaluación del AVA y las competencias adquiridas.

\section{RESULTADOS Y DISCUSIÓN}

Las actividades que se realizaron para el desarrollo del proyecto están descritas a continuación:

En primer lugar, se inició con un diagnóstico, donde se analiza la información relacionada con los diferentes teóricos sobre el tema de competencias docentes; se toma como referencia la opinión de los siguientes teóricos: Zabalza \& Arnau (2008), Braslavky (1999), Frade (2008), Monereo \& Pozo (2001), Perrenoud (2011), Beillerot (1998), y en cuanto a proyectos realizados se tienen los proyectos Tuning (2006), Reflex \& Proflex (2004) y el proyecto de Uniguajira llamado "Etnoeducación, Interculturalidad y Diversidad” (2010). Se realiza una comparación de los teóricos sobre competencias docentes para identificar las competencias que más se adapten al programa de Licenciatura en Etnoeducación y al contexto.

A continuación, se muestran dos cuadros comparativos de los teóricos y los proyectos sobre el concepto de competencias docentes:

\section{Figura 3.}

Comparativo de teóricos sobre el concepto de competencias docentes

\begin{tabular}{|c|c|c|c|c|c|c|}
\hline \multicolumn{7}{|c|}{ COMPARATIVO DE LOS TEORICOS SOBRE EL TEMA DE LAS COMPETENCIAS DOCENTES } \\
\hline AUTOR & $\begin{array}{l}\text { MIGUEL A. } \\
\text { ZABALZA }\end{array}$ & $\begin{array}{c}\text { CECILIA } \\
\text { BRASLAVSKY }\end{array}$ & LAURA FRADE & $\begin{array}{l}\text { MONEREO Y } \\
\text { POZO }\end{array}$ & $\begin{array}{c}\text { PHILIPPE } \\
\text { PERRENOUD }\end{array}$ & JACK BILLEROT \\
\hline $\begin{array}{l}\text { CONCEPTO DE } \\
\text { COMPETENCIA }\end{array}$ & $\begin{array}{l}\text { Las competencias } \\
\text { son las capacidades } \\
\text { o habilidades para } \\
\text { efectuar tareas o } \\
\text { hacer frente a } \\
\text { situaciones diversas } \\
\text { de forma eficaz en } \\
\text { un contexto } \\
\text { determinado, siendo } \\
\text { para ello necesario } \\
\text { movilizar actitudes, } \\
\text { habilidades y } \\
\text { conocimientos al } \\
\text { mismo tiempo y de } \\
\text { forma } \\
\text { interrelacionada. }\end{array}$ & $\begin{array}{l}\text { Competencia es un } \\
\text { saber hacer con } \\
\text { saber y con } \\
\text { concienciarespecto } \\
\text { del impacto de ese } \\
\text { hacer. También son } \\
\text { las habilidades } \\
\text { vinculadas con el } \\
\text { desempeño } \\
\text { autónomo, el } \\
\text { conocimiento } \\
\text { aplicado y aplicable, } \\
\text { el conocimiento en } \\
\text { acción, el saber } \\
\text { resultante de saber } \\
\text { hacer y saber } \\
\text { explicar lo que se } \\
\text { hace. }\end{array}$ & $\begin{array}{l}\text { Competencia es la } \\
\text { capacidad } \\
\text { adaptativa, cognitiva } \\
\text { y conductual para } \\
\text { responder } \\
\text { adecuadamente a } \\
\text { las demandas que } \\
\text { se presentan en el } \\
\text { entorno. } \\
\text { Frade define las } \\
\text { competencias desde } \\
\text { la perspectiva de las } \\
\text { inteligencias } \\
\text { múltiples y señala } \\
\text { una } \\
\text { inteligencia nlamada } \\
\text { Inteligencia } \\
\text { Educativa } \\
\text { (capacidad de } \\
\text { educar a los demás } \\
\text { en un momento } \\
\text { histórico } \\
\text { determinado de } \\
\text { manera adecuada a } \\
\text { las demandas que } \\
\text { se producen en el } \\
\text { entorno. }\end{array}$ & $\begin{array}{l}\text { Las competencias } \\
\text { son un repertorio } \\
\text { de estrategias } \\
\text { coordinadas para } \\
\text { resolver una } \\
\text { demanda } \\
\text { específica } \\
\text { correspondiente a } \\
\text { algún contexto } \\
\text { habitual (educativo, } \\
\text { familiar, } \\
\text { profesional, } \\
\text { personal) de la } \\
\text { actividad humana }\end{array}$ & $\begin{array}{l}\text { El concepto de } \\
\text { competencia } \\
\text { representa la } \\
\text { capacidad de } \\
\text { movilizar varios } \\
\text { recursos } \\
\text { cognitivos para } \\
\text { hacer frente a un } \\
\text { tipo } \\
\text { situaciones. }\end{array}$ & $\begin{array}{l}\text { El concepto de } \\
\text { competencias está } \\
\text { relacionado con } \\
\text { las situaciones de } \\
\text { aprendizaje. }\end{array}$ \\
\hline
\end{tabular}




\section{Figura.4.}

Comparativo de proyectos sobre el concepto de competencias docentes

\begin{tabular}{|c|c|c|c|}
\hline PROYECTO & PROYECTO TUNING & $\begin{array}{l}\text { PROYECTO } \\
\text { REFLEX Y } \\
\text { PROFLEX }\end{array}$ & $\begin{array}{l}\text { UNIGUAJIRA- } \\
\text { "ETNOEDUCACION, } \\
\text { INTERCULTURALIDAD } \\
\text { Y DIVER SIDAD" }\end{array}$ \\
\hline $\begin{array}{l}\text { CONCEPTO DE } \\
\text { COMPETENCIA }\end{array}$ & $\begin{array}{l}\text { Tuning define la Competencia como una combinación dinámica de } \\
\text { atributos, en relación a procedimientos, habilidades, actitudes y } \\
\text { responsabilidades, que describen los encargados del aprendizaje } \\
\text { de un programa educativo o lo que los alumnos son capaces de } \\
\text { demostrar al final de un proceso educativo. }\end{array}$ & $\begin{array}{l}\begin{array}{l}\text { Reflex y Proflex } \\
\text { definen }\end{array} \text { las } \\
\text { competencias como } \\
\text { las aptitudes, } \\
\text { destrezas } r \text { y } \\
\text { capacidades de los } \\
\text { graduados ren } \\
\text { educación superior y } \\
\text { que contribuyen a } \\
\text { aumentar la } \\
\text { productividad desde } \\
\text { una perspectiva } \\
\text { multidimensional. }\end{array}$ & $\begin{array}{l}\text { Las competencias sirven } \\
\text { para daruna dimensión a } \\
\text { los conocimientos, a los } \\
\text { valores y a las actitudes } \\
\text { básicas, esenciales para } \\
\text { la adquisición de } \\
\text { habilidades y } \\
\text { experiencias, a los logros } \\
\text { e indicadores que } \\
\text { apoyan al estudiante en } \\
\text { la definición de sus } \\
\text { propósitosy aspiraciones } \\
\text { y están dirigidos al } \\
\text { objetivo que permiten } \\
\text { que la información } \\
\text { construida a partir de } \\
\text { ellos sea confiable } \\
\text { completa. }\end{array}$ \\
\hline
\end{tabular}

Fuente: Elaboración Propia

Al mismo tiempo, se diseñó un instrumento que permitió evaluar el nivel de importancia de las competencias seleccionadas en la labor etnoeducativa y el nivel de desarrollo de la competencia que has alcanzado durante tus estudios. Con respecto a los resultados arrojados en el cuestionario, se diseñó una propuesta sobre las competencias y las unidades de competencias que los maestros en formación fortalecerán para su crecimiento pedagógico.

Los resultados de la aplicación del instrumento arrojan los diferentes niveles de importancia y desarrollo que consideran deben alcanzar los estudiantes. Se infiere que la importancia de la competencia está relacionada con lo que ellos consideran el desarrollo que a nivel pedagógico deben tener. Se debe hacer mucho énfasis en preparar los docentes en competencias relacionadas con el área tecnológica, el área cultural brindando alcances en los procesos de diversidad e interculturalidad, que son los factores que brindan un valor agregado al programa y en especial al proyecto de investigación. También se considera pertinente que el área didáctica debe desarrollarse en gran escala, ya que brinda la oportunidad de conocer y crear diversas estrategias, metodologías y procesos de evaluación que permiten innovar en el quehacer diario.

Un resultado que es sorprendente es el alto porcentaje de estudiantes que opinan sobre las competencias lingüísticas, en especial el dominio del idioma inglés, el 34,6\% consideran que no tiene ninguna importancia aprender o dominar dicho idioma; además, el 34\% opinan que el dominar la lengua wayuunaiki tiene un nivel medio de importancia.

En cuanto a las competencias específicas, las tres más importantes y que además los estudiantes consideran es la que se debe desarrollar en gran medida es la elaboración y utilización de materiales didácticos pertenecientes al contexto; el gestionar con responsabilidad su desarrollo personal y profesional y el diseñar estrategias de enseñanza-aprendizaje según contextos.

Después se continuó con el diseño y la planificación del proceso para ello se inició con selección de la metodología de trabajo para el diseño del AVA, se eligió la Metodología PACIE que permite el uso de herramientas tecnológicas para facilitar la interacción bajo la premisa de "Aprender Haciendo" y construir conocimiento de forma colaborativa. PACIE son las iníciales que comprenden las fases de implementación: Presencia-Alcance-Capacitación-Interacción-Elearning y en relación a la estructura de las aulas virtuales esta metodología se divide en 3 Bloques llamados: Bloque 0: Bloque PACIE que comprende la parte operativa del curso donde ocurren procesos de interacción y de construcción del conocimiento. El Bloque 1: Bloques Académicos que posee la información y el contenido del curso y los documentos a compartir y por último el Bloque 2: Bloque de Cierre está destinado a generar procesos de retroalimentación. 
Se prosigue con el diseño de la plataforma virtual, en un sitio web gratuito llamado gnomio basado en Moodle que contiene un espacio ilimitado para diseñar cursos virtuales, y que se encuentra en la siguiente dirección web: https:// www.gnomio.com/. El sitio donde se alojará el diplomado sobre competencias docentes para los maestros en formación del Programa de Licenciatura en Etnoeducación es: https://didactics.gnomio.com/. Dentro de la plataforma en el área de información se encuentra un módulo descriptivo y grafico sobre el manejo de la misma.

\section{Figura.5.}

Pantalla principal del Campus Virtual

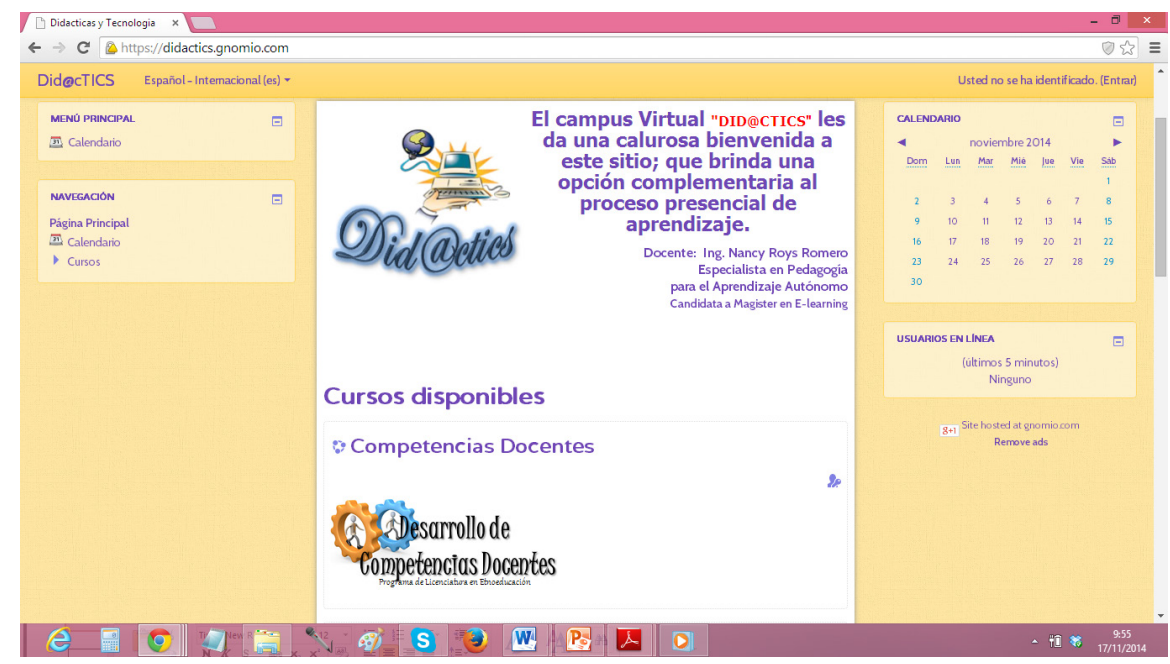

Fuente: Elaboración Propia

El esquema interno del curso, como se dijo anteriormente, está estructurado por Bloques, donde cada uno de ellos agrupa unas secciones o Áreas que complementan de manera detallada el proceso de aprendizaje, a través de la metodología PACIE.

\section{Figura.6.}

Estructura interna del curso

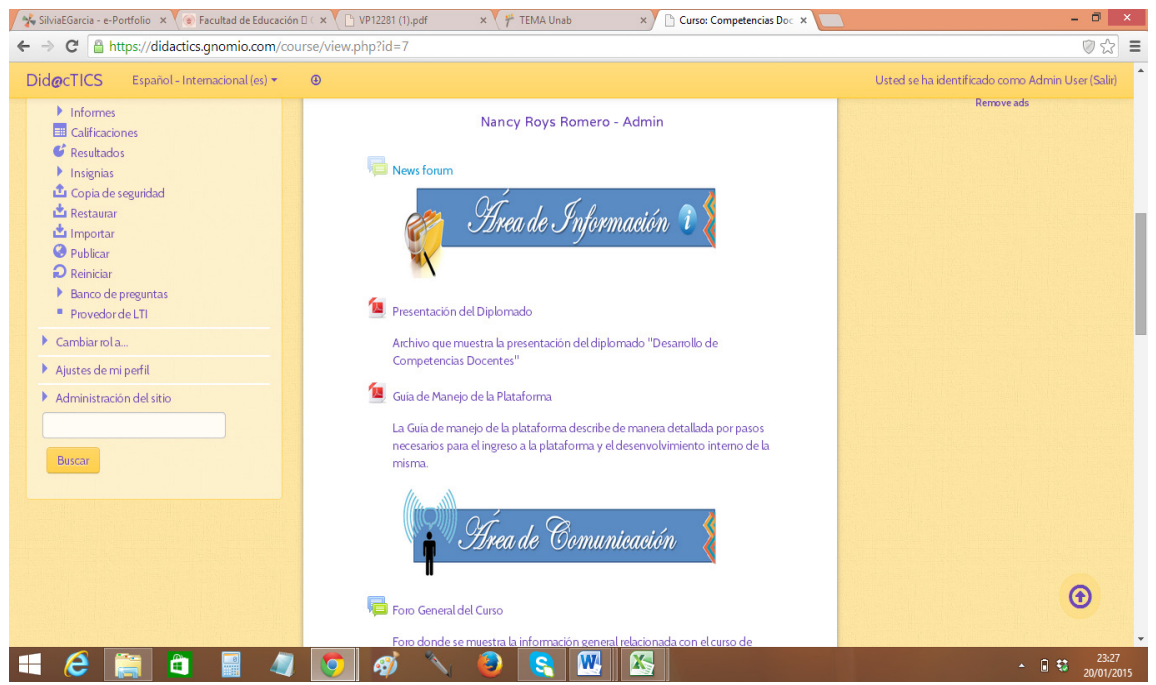

Fuente: Elaboración Propia 
También se diseñó un plan docente que describe a través de una dimensión teórica y conceptual la estructura del diplomado, está dirigido a maestros en formación del programa de Licenciatura en Etnoeducación que están interesados a conocer y fortalecer las competencias docentes que deben tener como etnoeducadores para asumir los nuevos roles en su labor pedagógica, a través de la integración de las TIC. El diplomado está diseñado por módulos; para el desarrollo de las lecciones de aprendizaje se utilizó el Programa eXeLearning, herramienta de autor de código abierto que permite la creación y publicación de contenidos; además de diversos programas de la Web 2.0 que ayudan a crear actividades interactivas y didácticas que motiven el proceso de aprendizaje.

Por último, se diseñó y aplico el instrumento final sobre la Evaluación del AVA y las competencias adquiridas, está dividido en dos grandes secciones: una trata sobre la evaluación del AVA y la otra es un diagnóstico sobre el grado de adquisición de las competencias docentes, este fue aplicado a la muestra seleccionada, y al final se realizó el análisis de los resultados.

Para realizar el proceso de evaluación del AVA, se utilizaron las áreas desarrolladas en la Guía de Evaluación para cursos virtuales de formación continua formulada por Rubio, Morocho, Torres \& Maldonado (2009), estas son: Tecnología, Formación, Diseño Instruccional y, por último, Servicios y Soporte.

El análisis de los resultados de la evaluación del AVA son los siguientes:

El análisis general del Área Tecnológica incluye los siguientes elementos: La infraestructura tecnológica tiene un $7 \%$ en su opción satisfactorio y un $93 \%$ en la opción muy satisfactorio; sigue la disponibilidad, rendimiento y capacidad un $4 \%$ en la opción satisfactorio y un $96 \%$ en la opción muy satisfactorio; luego, el elemento seguridad con un porcentaje del $38 \%$ en la opción satisfactorio y un $56 \%$ en la opción muy satisfactorio; continua el elemento accesibilidad con un 100\% en la opción muy satisfactorio; prosigue el elemento usabilidad y navegabilidad con un $7 \%$ en la opción satisfactorio y $93 \%$ en la opción muy satisfactorio y, finalmente, el elemento mantenimiento con un $23 \%$ en la opción satisfactorio y un $74 \%$ en la opción muy satisfactorio. Esto nos permite deducir que en esta área el AVA cumple con los requerimientos necesarios para identificar las tecnologías necesarias y garantizar la disponibilidad, la capacidad de almacenamiento, la seguridad y privacidad de los datos, el acceso a todos los estudiantes al curso virtual.

El análisis general del Área Formación que incluye Equipo docente y Alumnos, tiene un nivel muy satisfactorio del $100 \%$, lo que permite deducir que los estudiantes y docentes participantes del diplomado logran formarse en competencias con la utilización de medios tecnológicos.

El análisis general del Área Diseño Instruccional, que incluye los siguientes parámetros: Orientaciones generales del curso (100\%), objetivos $(97 \%)$, contenidos $(96 \%)$, interacción (96\%), seguimiento y tutoría (93\%) y por ultimo evaluación (100\%); todos estos con una escala de muy satisfactorio. Estos resultados nos permiten analizar los buenos resultados a nivel instruccional del diseño del AVA.

El AVA formula orientaciones claras y precisas sobre el diplomado y cuenta con contenidos enfocados y relacionados con el tema y con los objetivos propuestos, además de ofrecer recursos tecnológicos para llevar a cabo un proceso de interacción activo con los actores implicados en el proceso, realiza orientaciones de manera continua a los estudiantes participantes, además dispone de sistema fiable para evaluación en función de los objetivos

El análisis general del Área Servicios y Soporte que incluye los siguientes parámetros: Servicio de Información (93\%) y Atención al Alumno $(100 \%)$ con un nivel de muy satisfactorio en las preguntas realizadas a los estudiantes.

Estos resultados nos permiten deducir que el AVA cuenta con información general sobre los procesos para la realización del diplomado; además en relación con la atención al estudiante el AVA cuenta con manuales, recursos tecnológicos para la comunicación con las tutorías y por último la 
disponibilidad del servicio virtual durante las 24 horas del día. Todos estos parámetros garantizan la calidad del AVA a través del buen manejo de información y de servicio.

En cuanto a la segunda sesión del instrumento, enfocada a la adquisición de competencias, los resultados de las actividades que aparecen en el campus virtual (aplicaciones a situaciones problemas, manejos de conceptos, actividades, etc.) y la opinión de los mismos estudiantes en cuanto al análisis de todas las competencias trabajadas nos permiten deducir que las competencias que los estudiantes más fortalecieron en un nivel muy alto son las competencias Interpersonales (73\%), las competencias interculturales $(90 \%)$, las competencias tecnológicas ( $95 \%$ ), las competencias metacognitivas ( $92 \%$ ), las competencias evaluativas $(72 \%)$ y las competencias culturales (94\%); en un nivel alto encontramos las competencias didácticas (67\%), las competencias disciplinares (64\%) y las competencias investigativas (51\%). Después de analizar los resultados obtenidos en la sección sobre adquisición de competencias, se puede decir, que tanto el objetivo general como los objetivos específicos propuestos en la investigación se cumplen en su totalidad e igualmente se da respuesta a la pregunta problema. Se obtiene, por consiguiente, un gran impacto en la comunidad educativa a través de la utilización del AVA.

A continuación, se describe la importancia del fortalecimiento de las competencias trabajadas en el Diplomado:

- Competencias Interpersonales. Esta competencia fortalece en los estudiantes la capacidad del contacto con otros para mejor los procesos de comunicación.

- Competencias Didácticas. Esta competencia permitió fortalecer en los estudiantes la capacidad de preparar, construir y enseñar una clase, y a la vez utilizar estrategias para su correcto desarrollo.

- Competencias Disciplinares. Esta competencia permitió que los estudiantes fortalecieran sus nociones mínimas de conocimientos y contenidos para desarrollarse de manera eficaz en contextos diversos,
- Competencias Interculturales. El fortalecimiento de esta competencia es muy importante, ya que los estudiantes en su mayoría son personas pertenecientes a la etnia Wayuu, por lo tanto, desarrollaron habilidades y conocimientos enfocadas al desenvolvimiento adecuados de situaciones caracterizadas por la diversidad y la pluriculturalidad.

- Competencias Tecnológicas. Esta competencia desarrolló en los estudiantes habilidades enfocadas a la gestión de recursos tecnológicos para un mejor quehacer pedagógico.

- Competencias Metacognitivas. Permitió que el estudiante fortaleciera su capacidad de continuar aprendiendo y las diversas maneras en que puede hacerlo.

- Competencias Investigativas. El fortalecimiento de esta competencia permite transformar y generar nuevos conocimientos con el fin de investigar. Tiene como enfoque aprender a desarrollar proyectos de investigación.

- Competencias Evaluativas. Esta competencia permitió que los estudiantes ampliaran sus conocimientos acerca que es la evaluación, los tipos de evaluación que existe y de qué manera verificar el progreso en los estudiantes y de regular su proceso.

- Competencias Culturales. Esta competencia permitió conocer las creencias, valores de la cultura Wayuu, para poder trabajar de una manera más eficaz en su contexto.

Como resultado global del proyecto se espera impactar en un corto tiempo a los maestros en formación del Programa de Licenciatura en Etnoeducación a través del ambiente virtual creado https://didactics.gnomio.com que les permite fortalecer sus competencias docentes relacionadas en atender la diversidad étnica cultural de la población para mejorar su quehacer pedagógico y formar etnoeducadores de la más alta calidad y comprometidos con la generación de aprendizaje.

\section{CONCLUSIÓN}

El trabajo de investigación está basado en el diseño e implementación de un AVA para el fortalecimiento de las competencias docentes 
de maestros en formación del Programa de Licenciatura en Etnoeducación. El AVA es una opción y un complemento en la educación que facilitó el aprendizaje a través de módulos conceptuales y prácticos sobre las competencias genéricas y específicas que deben tener los etnoeducadores para su praxis pedagógica y favorece mejor la adaptación y el interés por aprender. También se integraron herramientas asincrónicas y sincrónicas que favorecieron de manera significativa el trabajo colaborativo de los estudiantes y espacios de interacción, foros y chats. Además, se incluyeron guías didácticas fundamentales para la realización de las actividades dentro de entorno virtual y rubricas para la valoración de las actividades.

En vista de lo anterior, y después de implementado el AVA en los estudiantes, hemos llegado a las siguientes conclusiones y recomendaciones:

- El AVA diseñado permite un proceso de construcción de conocimiento basado en competencias para el desarrollo de competencias docentes enfocadas a etnoeducadores.

- El proyecto analiza los efectos de la utilización de la tecnología y el resultado es que fortalece los procesos de aprendizaje de los estudiantes de Licenciatura en Etnoeducación.

- Los AVA fomentan el aprendizaje autónomo, donde el alumno se convierte en autor y protagonista de su proceso de aprendizaje.

- Se sugiere implementar el AVA en los demás estudiantes como un curso cambianteoptativo que le permita a toda la población estudiantil del programa de Licenciatura en Etnoeducación acceder y aprovechar los beneficios del diplomado; además es una manera de que los maestros puedan desarrollar competencias que los ayude a mejorar el rendimiento de las pruebas SABER PRO.

\section{REFERENCIAS BIBLIOGRÁFICAS}

Beillerot, J. (1998). La formación de formadores (entre la teoría y la práctica). Buenos Aires: Argentina. Ediciones Novedades Educativas.

Boneu, J. (2007). Plataformas abiertas de e-learning para el soporte de contenidos educativos abiertos. Revista de Universidad y Sociedad del Conocimiento, 4(1). Recuperado de http:// www.uoc.edu/rusc/4/1/dt/esp/boneu.pdf

Díaz Osorio, G. Gutiérrez Peraza, L. Merlano

Blanco, C. Díaz Morales, A. (2010). Una educación para el desarrollo de competencias. Recuperado de https://ingcarlosmerlano.files. wordpress.com/2010/05/una-educacion-parael-desarrollo-de-competencias.pdf

Frade, L. (2008). Evaluación y Perspectivas en las competencias docentes. Recuperado de http://www.calidadeducativa.com/ articulos-de-interes/laura-frade/2008/ evaluacion-y-perspectivas-en-las-competencias-docentes.html

Ministerio de Educación Nacional -MEN. (2006). Documento $N^{\circ} 3$. Estándares Básicos de Competencia en Lenguaje, Matemáticas, Ciencias y Ciudadanas. Bogotá: MEN. Recuperado de http://www.mineducacion. gov.co/1621/articles-116042_archivo_pdf.pdf

Monereo, C. Pozo, J. (2001). Decálogo para el futuro. Cuaderno de pedagogía. 48 (298). 49- 78

Perrenoud, P. (2011). Diez nuevas competencias para enseñar. Invitacion al viaje. Bogota, Colombia: Grao.

Ricardo, C. (2013). Formacion y desarrollo de la competencia intercutural en Ambientes Virtuales de Aprendizaje. Tesis Doctoral. Universidad Nacional de Educacion a Distancia UNED. España.

Rubio, M., Morocho, M., Torres, J., Maldonado R, J., Ramirez, I. (2009). Guia de Evaluacion para cursos virtuales de formacion continua. Basada en el marco regulador para la oferta de cursos virtuales del proyecto Centro Virtual para el desarrollo de estandares de calidad para la Educacion Superior a distancia en America Latina y el Caribe.

Sanabria, L., Macias, D. (2006). Formacion de competencias docentes: diseñar y aprender con ambientes computacionales.Universidad Pedagogica Nacional. Colciencias.

Universidad de La Guajira. Etnoeducacion, Interculturalidad y Diversidad. Programa de licenciatura en etnoeducacion para la basica primaria: Documento maestro para la actualizacion del registro calificado. Bogota, Colombia: Editorial Gente Nueva. 
Velásquez, A., López, E. (2011). Una propuesta metodológica para contribuir al desarrollo de competencias investigativas en la Educación Científica a partir del diseño de una unidad didáctica construida sobre fundamentos de la alfabetización científica y tecnológica. Praxis.
7. p.p 42-51. Recuperado de http://revistas. unimagdalena.edu.co/index.php/praxis/ article/view/7

Zabalza, A., Arnau, L. (2008). 11 Ideas clave: como aprender y enseñar competencias. Barcelona España: Ed. Graó. 